\title{
Connection between Schinzel's conjecture and divisibility of the class number $h_{p}^{+}$
}

\author{
by
}

\author{
Stanislav Jakubec (Bratislava)
}

\section{Notations}

- $(2 m+1) ! !=1 \cdot 3 \cdot 5 \cdot \ldots \cdot(2 m+1)$,

- $h_{p}^{+}$— class number of the field $\mathbb{Q}\left(\zeta_{p}+\zeta_{p}^{-1}\right)$,

- $Q_{2}=\left(2^{q-1}-1\right) / q$,

- $A_{j}=1+1 / 2+\ldots+1 / j, \quad A_{0}=0$,

- $B_{k}, B_{k}(X)$-Bernoulli number and Bernoulli polynomial,

- $E_{k}, E_{k}(X)$-Euler number and Euler polynomial.

Introduction. The aim of this paper is to prove the following

Theorem 1. Let $p=8 k(2 m+1) ! !-1$ be a prime with the property that $l=4 k(2 m+1) ! !-1$ and $2 k(2 m+1) ! !-1$ are primes. Then $\left(h_{p}^{+},(2 m+1) ! !\right)=1$.

It is easy to observe a connection between Theorem 1 and Schinzel's conjecture for the linear polynomials $8 X(2 m+1) ! !-1,4 X(2 m+1) ! !-1$ and $2 X(2 m+1) ! !-1$. If Schinzel's conjecture for the linear polynomials holds, then there are infinitely many prime numbers $p$ which satisfy the assumptions of Theorem 1.

SChinZeL's COnJECture. Let $s \geq 1$, let $f_{1}(X), \ldots, f_{s}(X)$ be irreducible polynomials with integral coefficients; assume that the leading coefficient of $f_{i}(X)$ is positive and that no integer $n>1$ divides all the numbers $f_{1}(m) f_{2}(m) \ldots f_{s}(m)$. Then there exists one (and then, as may be proved, necessarily infinitely many) natural number(s) $m$ such that $f_{1}(m), \ldots, f_{s}(m)$ are all primes.

2000 Mathematics Subject Classification: Primary 11R29; Secondary 11B68. 
The proof of Theorem 1 will be based on Theorem 5 of [2]. Let $j$ be an integer, $0<j<2 q, j \equiv 0(\bmod 2)$. Define

$$
S_{j}=\sum_{i=1}^{(q-1) / 2} A_{i} \sum_{\substack{k=1 \\ k \equiv 1(\bmod 2) \\ 2 j i \neq-k(\bmod q)}}^{j-1} \frac{1}{2 j i+k}-\sum_{i=(q+1) / 2}^{q-1} A_{i} \sum_{\substack{k=1 \\ k \equiv 1(\bmod 2) \\ 2 j i \neq-k(\bmod q)}}^{j-1} \frac{1}{2 j i+k}
$$

Theorem (5 of [2]). Let $q$ be an odd prime. Let $l, p$ be primes such that $p=2 l+1, l \equiv 3(\bmod 4), p \equiv-1(\bmod q)$, and let the order of the prime $q$ modulo $l$ be $(l-1) / 2$. Suppose that for each $j$ such that $S_{j} \equiv 0$ $(\bmod q)$ there exists $n,(n, 2 q)=1, n \mid p+1$, such that $S_{j^{*}} \not \equiv 0(\bmod q)$, where $j^{*} \equiv n j(\bmod 2 q)$. Then $q$ does not divide $h_{p}^{+}$, the class number of the real cyclotomic field $\mathbb{Q}\left(\zeta_{p}+\zeta_{p}^{-1}\right)$.

LEMMA 1. If $q>3$, then $S_{j} \equiv-Q_{2}-\sum_{k=1}^{(q-3) / 2} \frac{\left(2^{2 k}-1\right)\left(2^{2 k+1}-1\right)}{2 k \cdot 2^{2 k}}\left(\begin{array}{c}q-1 \\ 2 k\end{array}\right) j^{2 k} B_{2 k} B_{q-1-2 k}(\bmod q)$.

Pr o o f. Consider the sums $\sum_{i=1}^{(q-1) / 2}(a+i)^{q-2} A_{i}$ modulo $q$, where $a \in \mathbb{Q}$, $a \not \equiv 0(\bmod q)$ and $a$ is a $q$-integer.

Define $S_{n}(i)=1^{n}+2^{n}+\ldots+(i-1)^{n}$ for $n \geq 0$. The following formula will be used:

$$
S_{n}(i)=\frac{1}{n+1}\left(B_{n+1}(i)-B_{n+1}\right) .
$$

The binomial theorem applied to $(a+i)^{q-2}$ yields

$$
\begin{aligned}
\sum_{i=1}^{(q-1) / 2}(a+i)^{q-2} A_{i} \equiv & A_{(q-1) / 2} \sum_{n=0}^{q-2}\left(\begin{array}{c}
q-2 \\
n
\end{array}\right) a^{q-2-n} S_{n}\left(\frac{q+1}{2}\right) \\
& -\sum_{n=0}^{q-2}\left(\begin{array}{c}
q-2 \\
n
\end{array}\right) a^{q-2-n} \sum_{i=1}^{(q-1) / 2} \frac{1}{i} S_{n}(i)(\bmod q) .
\end{aligned}
$$

Since

$S_{n}\left(\frac{q+1}{2}\right) \equiv \frac{1}{n+1}\left(B_{n+1}\left(\frac{1}{2}\right)-B_{n+1}\right) \equiv \frac{1}{n+1}\left(2^{-n}-2\right) B_{n+1}(\bmod q)$ for $0<n \leq q-2$, it follows that

$$
\begin{aligned}
& \sum_{n=0}^{q-2}\left(\begin{array}{c}
q-2 \\
n
\end{array}\right) a^{q-2-n} S_{n}\left(\frac{q+1}{2}\right) \\
& \equiv-\frac{1}{a}+\sum_{n=0}^{q-2}\left(\begin{array}{c}
q-2 \\
n
\end{array}\right) a^{q-2-n} \frac{1}{n+1}\left(2^{-n}-2\right) B_{n+1}(\bmod q)
\end{aligned}
$$


Note that $\left(2^{q-1}-1\right) B_{q-1}$ is a $q$-integer; in fact $\left(2^{q-1}-1\right) B_{q-1} \equiv-Q_{2}$ $(\bmod q)$.

From the congruence

$$
\left(\begin{array}{c}
q-2 \\
n
\end{array}\right) \equiv-\left(\begin{array}{c}
q-1 \\
n+1
\end{array}\right)(n+1) \equiv(-1)^{n}(n+1)(\bmod q),
$$

we get

$$
\begin{aligned}
\sum_{n=0}^{q-2}\left(\begin{array}{c}
q-2 \\
n
\end{array}\right) a^{q-2-n} S_{n}\left(\frac{q+1}{2}\right) \equiv & -\frac{1}{a}-\sum_{k=1}^{q-1}\left(\begin{array}{c}
q-1 \\
k
\end{array}\right) a^{-k}\left(2^{1-k}-2\right) B_{k} \\
\equiv & -\frac{1}{a}-2 \sum_{k=1}^{q-1}\left(\begin{array}{c}
q-1 \\
k
\end{array}\right)\left(\frac{1}{2 a}\right)^{k} B_{k} \\
& +2 \sum_{k=1}^{q-1}\left(\begin{array}{c}
q-1 \\
k
\end{array}\right)\left(\frac{1}{a}\right)^{k} B_{k}(\bmod q) .
\end{aligned}
$$

Using the formula ((50.5.33) of [1])

$$
\sum_{k=0}^{n} \frac{(-n)_{k}}{k !} a^{k} B_{k}(X)=\sum_{k=0}^{n}(-1)^{k}\left(\begin{array}{l}
n \\
k
\end{array}\right) a^{k} B_{k}(X)=(-a)^{n} B_{n}\left(X-\frac{1}{a}\right),
$$

we altogether get

$$
\begin{aligned}
A_{(q-1) / 2} \sum_{n=0}^{q-2} & \left(\begin{array}{c}
q-2 \\
n
\end{array}\right) a^{q-2-n} S_{n}\left(\frac{q+1}{2}\right) \\
& \equiv A_{(q-1) / 2}\left(2 B_{q-1}(a)-2 B_{q-1}(2 a)-2 Q_{2}-\frac{1}{a}\right)(\bmod q) .
\end{aligned}
$$

Using the same procedure we get the following congruences modulo $q$ :

$$
\begin{aligned}
& \sum_{n=0}^{q-2}\left(\begin{array}{c}
q-2 \\
n
\end{array}\right) a^{q-2-n} \sum_{i=1}^{(q-1) / 2} \frac{1}{i} S_{n}(i) \\
& \equiv \frac{1}{a} \sum_{i=1}^{(q-1) / 2} \frac{i-1}{i} \\
&+\sum_{n=1}^{q-2}(-1)^{n}(n+1) \frac{1}{a^{n+1}} \sum_{i=1}^{(q-1) / 2} \frac{1}{i} \cdot \frac{1}{n+1}\left(B_{n+1}(i)-B_{n+1}\right) \\
& \equiv \frac{1}{a}\left(\frac{q-1}{2}-A_{(q-1) / 2}\right)+\sum_{k=2}^{q-1}(-1)^{k+1} \frac{1}{a^{k}} \sum_{i=1}^{(q-1) / 2} \frac{1}{i}\left(B_{k}(i)-B_{k}\right)
\end{aligned}
$$




$$
\begin{aligned}
\equiv & \frac{1}{a}\left(\frac{q-1}{2}-A_{(q-1) / 2}\right)-\frac{1}{a} \sum_{i=1}^{(q-1) / 2} \frac{1}{i}\left(B_{1}(i)-B_{1}\right) \\
& -\sum_{k=0}^{q-1}(-1)^{k}\left(\begin{array}{c}
q-1 \\
k
\end{array}\right)\left(\frac{-1}{a}\right)^{k} \sum_{i=1}^{(q-1) / 2} \frac{1}{i}\left(B_{k}(i)-B_{k}\right) .
\end{aligned}
$$

By switching the order of summation in the sum

$$
\sum_{k=0}^{q-1}(-1)^{k}\left(\begin{array}{c}
q-1 \\
k
\end{array}\right)\left(\frac{-1}{a}\right)^{k} \sum_{i=1}^{(q-1) / 2} \frac{1}{i}\left(B_{k}(i)-B_{k}\right),
$$

using the above quoted formula (50.5.33) of [1] we get

$$
\begin{aligned}
\sum_{n=0}^{q-2}\left(\begin{array}{c}
q-2 \\
n
\end{array}\right) a^{q-2-n} & \sum_{i=1}^{(q-1) / 2} \frac{1}{i} S_{n}(i) \\
& \equiv-\frac{1}{a} A_{(q-1) / 2}-\sum_{i=1}^{(q-1) / 2} \frac{1}{i}\left(B_{q-1}(i+a)-B_{q-1}(a)\right)(\bmod q) .
\end{aligned}
$$

Altogether we get

$$
\text { (1) } \begin{aligned}
\sum_{i=1}^{(q-1) / 2}(a+i)^{q-2} A_{i} \equiv & 2 A_{(q-1) / 2}\left(B_{q-1}(a)-B_{q-1}(2 a)-Q_{2}\right) \\
& +\sum_{i=1}^{(q-1) / 2} \frac{1}{i}\left(B_{q-1}(i+a)-B_{q-1}(a)\right)(\bmod q) .
\end{aligned}
$$

The congruence $(1)$ is thus proved for $a \not \equiv 0(\bmod q)$. We now prove it for $a \equiv 0(\bmod q)$. That is, for $a \equiv 0(\bmod q)$ we claim that

$$
\sum_{i=1}^{(q-1) / 2} \frac{1}{i} A_{i} \equiv-2 A_{(q-1) / 2} Q_{2}+\sum_{i=1}^{(q-1) / 2} \frac{1}{i}\left(B_{q-1}(i)-B_{q-1}\right)(\bmod q) .
$$

Because $\sum_{i=1}^{(q-1) / 2} 1 / i^{2} \equiv 0(\bmod q)$, we have

$$
\sum_{i=1}^{(q-1) / 2} \frac{1}{i} A_{i} \equiv \sum_{i=1}^{(q-1) / 2} \frac{1}{i} A_{i-1} \equiv \frac{1}{q-1} \sum_{i=1}^{(q-1) / 2} \frac{1}{i}\left(B_{q-1}(i)-B_{q-1}\right)(\bmod q) .
$$

It is sufficient to prove

$$
\sum_{i=1}^{(q-1) / 2} \frac{1}{i} A_{i-1} \equiv-A_{(q-1) / 2} Q_{2} \equiv \frac{1}{2} A_{(q-1) / 2}^{2}(\bmod q) .
$$


This follows from the relation

$A_{(q-1) / 2}^{2}=1^{2}+\left(\frac{1}{2}\right)^{2}+\ldots+\left(\frac{1}{\frac{q-1}{2}}\right)^{2}+2\left(1 \cdot \frac{1}{2}+1 \cdot \frac{1}{3}+\ldots+\frac{1}{\frac{q-3}{2}} \cdot \frac{1}{\frac{q-1}{2}}\right)$.

Now put

$$
S^{*}=\sum_{i=1}^{(q-1) / 2}(i+a)^{q-2} A_{i}-\sum_{i=(q+1) / 2}^{q-1}(i+a)^{q-2} A_{i} .
$$

For the numbers $A_{0}, A_{1}, \ldots, A_{q-1}$ we have

$$
A_{(q-1) / 2+k} \equiv A_{(q-1) / 2-k}(\bmod q) \quad \text { for } k=1,2, \ldots,(q-1) / 2,
$$

and so

$$
\begin{aligned}
- & \sum_{i=(q+1) / 2}^{q-1}(i+a)^{q-2} A_{i} \\
\equiv & -\left(\frac{q-1}{2}+1+a\right)^{q-2} A_{(q-1) / 2-1} \\
& -\left(\frac{q-1}{2}+2+a\right)^{q-2} A_{(q-1) / 2-2}-\ldots-\left(\frac{q-1}{2}+\frac{q-3}{2}+a\right)^{q-2} A_{1} \\
\equiv & \left(\frac{q-3}{2}+1-a\right)^{q-2} A_{(q-3) / 2} \\
& +\left(\frac{q-5}{2}+1-a\right)^{q-2} A_{(q-5) / 2}+\ldots+(1+1-a)^{q-2} A_{1} \\
\equiv & \sum_{i=1}^{(q-1) / 2}(i+1-a)^{q-2} A_{i}-\left(\frac{1}{2}-a\right)^{q-2} A_{(q-1) / 2}(\bmod q) .
\end{aligned}
$$

By congruence (1) we have

$$
\begin{aligned}
S^{*} \equiv & 2 A_{(q-1) / 2}\left(B_{q-1}(a)-B_{q-1}(2 a)-Q_{2}\right) \\
& +\sum_{i=1}^{(q-1) / 2} \frac{1}{i}\left(B_{q-1}(a+i)-B_{q-1}(a)\right) \\
& +2 A_{(q-1) / 2}\left(B_{q-1}(1-a)-B_{q-1}(2-2 a)-Q_{2}\right) \\
& +\sum_{i=1}^{(q-1) / 2} \frac{1}{i}\left(B_{q-1}(1-a+i)-B_{q-1}(1-a)\right) \\
& -\left(\frac{1}{2}-a\right)^{q-2} A_{(q-1) / 2}(\bmod q) .
\end{aligned}
$$


Since $B_{q-1}(X)=B_{q-1}(1-X)$, we have $B_{q-1}(2-2 a)=B_{q-1}(2 a-1)$, $B_{q-1}(1-a+i)=B_{q-1}(a-i)$ and $B_{q-1}(1-a)=B_{q-1}(a)$. Moreover, the formula

$$
B_{q-1}(X)=B_{q-1}(-X)-(q-1) X^{q-2}
$$

gives

$$
\begin{aligned}
B_{q-1}(2 a-1) & =B_{q-1}(1-2 a)+2(q-1) B_{1}(2 a-1)^{q-2} \\
& =B_{q-1}(2 a)-(q-1)(2 a-1)^{q-2} .
\end{aligned}
$$

So we obtain

(2) $S^{*} \equiv 4 A_{(q-1) / 2}\left(B_{q-1}(a)-B_{q-1}(2 a)-Q_{2}\right)$

$$
+\sum_{i=1}^{(q-1) / 2} \frac{1}{i}\left(B_{q-1}(a+i)+B_{q-1}(a-i)-2 B_{q-1}(a)\right)(\bmod q) .
$$

Now using the congruence (2) let us start to compute the sums $S_{j}$ defined in the introduction. We write

$$
\begin{aligned}
& S_{j} \equiv \sum_{i=1}^{(q-1) / 2} A_{i} \sum_{\substack{k=1 \\
k \equiv 1(\bmod 2)}}^{j-1}(2 j i+k)^{q-2}-\sum_{i=(q+1) / 2}^{q-1} A_{i} \sum_{\substack{k=1 \\
k \equiv 1(\bmod 2)}}^{j-1}(2 j i+k)^{q-2} \\
& \equiv(2 j)^{q-2}\left(\sum_{i=1}^{(q-1) / 2} A_{i} \sum_{\substack{k=1 \\
k \equiv 1(\bmod 2)}}^{j-1}\left(i+\frac{k}{2 j}\right)^{q-2}\right. \\
&\left.-\sum_{i=(q+1) / 2}^{q-1} A_{i} \sum_{\substack{k=1 \\
k \equiv 1(\bmod 2)}}^{j-1}\left(i+\frac{k}{2 j}\right)^{q-2}\right)(\bmod q) .
\end{aligned}
$$

We now reverse the order of summations. For the inner summation (over $i$ ) we use (2) with $a=k /(2 j)$. For the summation over $k$, the following formulas will be used. According to (50.7.2) of [1],

$$
\sum_{k=0}^{n-1} B_{m}\left(X+\frac{k}{n}\right)=n^{1-m} B_{m}(n X),
$$

and by (50.7.4) of [1],

$$
\sum_{k=0}^{2 n-1}(-1)^{k+1} B_{m}\left(X+\frac{k}{2 n}\right)=2^{-m} n^{1-m} m E_{m-1}(2 n X) .
$$

Let us denote by $\sum^{\prime}$ a summation over odd values of $k$. In view of the congruence (2) it is necessary to compute

$$
\sum_{k=1}^{j-1}\left(B_{q-1}\left(\frac{k}{2 j}+i\right)+B_{q-1}\left(\frac{k}{2 j}-i\right)\right) .
$$


By the identity $B_{q-1}(X)=B_{q-1}(1-X)$ we have

$$
\sum_{k=1}^{j-1}\left(B_{q-1}\left(\frac{k}{2 j}+i\right)+B_{q-1}\left(\frac{k}{2 j}-i\right)\right)=\sum_{k=1}^{2 j-1} B_{q-1}\left(\frac{k}{2 j}+i\right) .
$$

Using (3) and (4), we altogether obtain

$$
\begin{aligned}
& \sum_{k=1}^{j-1}\left(B_{q-1}\left(\frac{k}{2 j}+i\right)+B_{q-1}\left(\frac{k}{2 j}-i\right)\right) \\
& \quad=\frac{1}{2}\left((2 j)^{1-(q-1)} B_{q-1}(2 j i)+(q-1) 2^{-(q-1)} j^{1-(q-1)} E_{q-2}(2 j i)\right) .
\end{aligned}
$$

Using the identity

$$
E_{q-2}(2 j i)=\frac{2}{q-1}\left(B_{q-1}(2 j i)-2^{q-1} B_{q-1}(j i)\right),
$$

we get

$$
\text { (5) } \begin{aligned}
\sum_{k=1}^{j-1}\left(B_{q-1}\left(\frac{k}{2 j}+i\right)+B_{q-1}\right. & \left.\left(\frac{k}{2 j}-i\right)\right) \\
& =j^{2-q}\left(2^{2-q} B_{q-1}(2 j i)-B_{q-1}(j i)\right) .
\end{aligned}
$$

Consider now the sums

$$
\sum_{k=1}^{j-1} B_{q-1}\left(\frac{k}{2 j}\right), \quad \sum_{k=1}^{j-1} B_{q-1}\left(\frac{k}{j}\right) .
$$

The identity (5) for $i=0$ implies

$$
\sum_{k=1}^{j-1} B_{q-1}\left(\frac{k}{2 j}\right)=\frac{1}{2} j^{2-q}\left(2^{2-q}-1\right) B_{q-1},
$$

and using (3) and (4) we obtain, since $j$ is even,

$$
\begin{aligned}
\sum_{k=0}^{j-1} B_{q-1}\left(\frac{k}{j}\right) & =j^{1-(q-1)} B_{q-1}, \\
\sum_{k=0}^{j-1}(-1)^{k+1} B_{q-1}\left(\frac{k}{j}\right) & =2^{-(q-1)}\left(\frac{j}{2}\right)^{1-(q-1)}(q-1) E_{q-2}(0) .
\end{aligned}
$$

By summing the last two identities using the previous formula for a relation between Euler and Bernoulli polynomials we have

$$
\sum_{k=1}^{j-1} B_{q-1}\left(\frac{k}{j}\right)=\left(1-2^{q-1}\right) 2^{1-q}\left(\frac{j}{2}\right)^{2-q} B_{q-1}+\frac{1}{2} j^{2-q} B_{q-1} .
$$


Using the formula for $S^{*}$ we get

$$
\begin{aligned}
S_{j} & \equiv 2^{q-1} A_{(q-1) / 2}\left(\left(2^{2-q}-1\right) B_{q-1}-\left(1-2^{q-1}\right) B_{q-1}-B_{q-1}-Q_{2}\right) \\
& +2^{q-2} \sum_{i=1}^{(q-1) / 2} \frac{1}{i}\left(\left(2^{2-q} B_{q-1}(2 i j)-B_{q-1}(i j)\right)-\left(2^{2-q}-1\right) B_{q-1}\right)(\bmod q) .
\end{aligned}
$$

This shows that if $q>3$ then

$$
S_{j} \equiv \sum_{i=1}^{(q-1) / 2} \frac{1}{i}\left(B_{q-1}^{*}(2 j i)-\frac{1}{2} B_{q-1}^{*}(j i)\right)(\bmod q),
$$

where $B_{q-1}^{*}(X)=B_{q-1}(X)-B_{q-1}$.

For $c \in \mathbb{Z}$, we have

$$
\begin{aligned}
\sum_{k=1}^{(q-1) / 2} \frac{1}{k} B_{q-1}^{*}(k c) & \equiv \sum_{k=1}^{(q-1) / 2} k^{q-2}\left(B_{q-1}(k c)-B_{q-1}\right) \\
& =\sum_{k=1}^{(q-1) / 2} k^{q-2} B_{q-1}(k c)-B_{q-1} \sum_{k=1}^{(q-1) / 2} k^{q-2} .
\end{aligned}
$$

Using the formula (50.7.14) of [1]:

$$
\sum_{k=1}^{n} k^{r} B_{m}(k X)=\sum_{k=0}^{m} \frac{(-m)_{k}}{(r+k+1) k !}(-X)^{k} B_{m-k}\left(B_{r+k+1}(n+1)-B_{r+k+1}\right),
$$

we have

$$
\begin{aligned}
& \sum_{k=1}^{(q-1) / 2} k^{q-2} B_{q-1}(k c) \\
& \quad=\sum_{k=0}^{q-1}\left(\begin{array}{c}
q-1 \\
k
\end{array}\right) \frac{(-1)^{k}}{q-1+k}(-c)^{k} B_{q-1-k}\left(B_{q-1+k}\left(\frac{q+1}{2}\right)-B_{q-1+k}\right) .
\end{aligned}
$$

For the term with $k=0$ on the right-hand side, note that

$$
\frac{1}{q-1}\left(B_{q-1}\left(\frac{q+1}{2}\right)-B_{q-1}\right)=\sum_{k=1}^{(q-1) / 2} k^{q-2} .
$$

Thus we have

$$
\begin{aligned}
& \sum_{k=1}^{(q-1) / 2} \frac{1}{k} B_{q-1}^{*}(k c) \\
& \equiv \sum_{k=1}^{q-1}\left(\begin{array}{c}
q-1 \\
k
\end{array}\right) \frac{1}{q-1+k} c^{k} B_{q-1-k}\left(B_{q-1+k}\left(\frac{q+1}{2}\right)-B_{q-1+k}\right)(\bmod q) .
\end{aligned}
$$


For $k=q-1$ we have

$\frac{1}{2(q-1)} c^{q-1} B_{0}\left(B_{2(q-1)}\left(\frac{q+1}{2}\right)-B_{2(q-1)}\right) \equiv \sum_{k=1}^{(q-1) / 2} \frac{1}{k} \equiv-2 Q_{2}(\bmod q)$,

hence

$$
\begin{aligned}
\sum_{k=1}^{(q-1) / 2} \frac{1}{k} B_{q-1}^{*}(k c) \equiv & -2 Q_{2}+\sum_{k=1}^{q-2}\left(\begin{array}{c}
q-1 \\
k
\end{array}\right) \frac{1}{q-1+k} \\
& \times c^{k} B_{q-1-k}\left(B_{q-1+k}\left(\frac{q+1}{2}\right)-B_{q-1+k}\right)(\bmod q)
\end{aligned}
$$

Clearly

$$
\begin{aligned}
B_{q-1+k}\left(\frac{q+1}{2}\right) & \equiv B_{q-1+k}\left(\frac{1}{2}\right)(\bmod q), \\
B_{q-1+k}\left(\frac{1}{2}\right) & =\left(2^{2-q-k}-1\right) B_{q-1+k} .
\end{aligned}
$$

By Kummer's congruence $B_{q-1+k} \equiv(q-1+k) B_{k} / k(\bmod q)$. Hence

$$
\begin{aligned}
& \text { (6) } \quad \sum_{k=1}^{(q-1) / 2} \frac{1}{k} B_{q-1}^{*}(k c) \\
& \equiv-2 Q_{2}+\sum_{k=1}^{(q-3) / 2}\left(\begin{array}{c}
q-1 \\
2 k
\end{array}\right) \frac{1}{2 k} c^{2 k}\left(\frac{2}{2^{2 k}}-2\right) B_{q-1-2 k} B_{2 k}(\bmod q) .
\end{aligned}
$$

Define

$$
F(X)=\sum_{k=1}^{(q-3) / 2}\left(\begin{array}{c}
q-1 \\
2 k
\end{array}\right) \frac{X^{2 k}}{2 k} B_{2 k} B_{q-1-2 k} .
$$

By (6) we have

$$
\sum_{k=1}^{(q-1) / 2} \frac{1}{k} B_{q-1}^{*}(k c) \equiv-2 Q_{2}+2 F\left(\frac{c}{2}\right)-2 F(c)(\bmod q) .
$$

Hence if $q>3$, then

$$
\begin{aligned}
S_{j} & \equiv \sum_{i=1}^{(q-1) / 2} \frac{1}{i}\left(B_{q-1}^{*}(2 j i)-\frac{1}{2} B_{q-1}^{*}(j i)\right) \\
& \equiv-2 Q_{2}+2 F\left(\frac{2 j}{2}\right)-2 F(2 j)-\frac{1}{2}\left(-2 Q_{2}+2 F\left(\frac{j}{2}\right)-2 F(j)\right) \\
& \equiv-Q_{2}+3 F(j)-2 F(2 j)-F\left(\frac{j}{2}\right)(\bmod q),
\end{aligned}
$$


hence

$$
S_{j} \equiv-Q_{2}-\sum_{k=1}^{(q-3) / 2} \frac{\left(2^{2 k}-1\right)\left(2^{2 k+1}-1\right)}{2 k \cdot 2^{2 k}}\left(\begin{array}{c}
q-1 \\
2 k
\end{array}\right) j^{2 k} B_{2 k} B_{q-1-2 k}(\bmod q) .
$$

Lemma 1 is proved.

Proof of Theorem 1. If $q=3$ then from [3] it follows that $\left(h_{p}^{+}, 3\right)=1$. Let $q$ be a prime, $3<q \leq 2 m+1$. By the quadratic reciprocity law we have

$$
\left(\frac{q}{l}\right)=\left(\frac{-1}{q}\right)\left(\frac{l}{q}\right)=\left(\frac{-1}{q}\right)\left(\frac{4 k(2 m+1) ! !-1}{q}\right)=\left(\frac{-1}{q}\right)\left(\frac{-1}{q}\right)=1 .
$$

The number $(l-1) / 2=2 k(2 m+1) ! !-1$ is prime, and so the order of $q$ modulo $l$ is $(l-1) / 2$. Let $j$ be such that $S_{j} \equiv 0(\bmod q)$. Now we use Theorem 5 of [2]. The number $p+1$ has divisors $n=1,3, \ldots, q-2$. For some $n$ we have $S_{n j} \not \equiv 0(\bmod q)$. To prove this, note that $S_{j}$ is a polynomial in $j$ of degree at most $q-3$. Suppose that $S_{j}$ has roots $j, 3 j, \ldots,(q-2) j$; then $-j,-3 j, \ldots,-(q-2) j$ are also roots of $S_{j}$. Thus the number of roots of $S_{j}$ is $q-1$, which is a contradiction.

To complete the proof it is necessary to prove that $S_{j}$ is non-zero modulo $q$. For this we shall use the bound for the first factor $h_{q}^{-}$from [4] and $[5]$,

$$
h_{q}^{-}<2 q\left(\frac{q}{24}\right)^{(q-1) / 4} .
$$

Let ii $(q)$ be the index of irregularity and $d$ be the order $(\bmod q)$ of 2 . If $S_{j}$ is a zero polynomial modulo $q$, then

$$
\frac{\left(2^{2 k}-1\right)\left(2^{2 k+1}-1\right)}{2 k \cdot 2^{2 k}}\left(\begin{array}{c}
q-1 \\
2 k
\end{array}\right) B_{2 k} B_{q-1-2 k} \equiv 0(\bmod q)
$$

for $k=1,2, \ldots,(q-3) / 2$, and then

$$
\operatorname{ii}(q)>\frac{1}{2}\left(\frac{q-3}{2}-2 \frac{q-1}{d}\right)>\frac{1}{2}\left(\frac{q-3}{2}-2 \frac{q-1}{\log _{2} q}\right) .
$$

This yields

and so

$$
q^{\frac{1}{2}\left(\frac{q-3}{2}-2 \frac{q-1}{\log _{2} q}\right)}<2 q\left(\frac{q}{24}\right)^{(q-1) / 4}
$$

$$
1<4 q^{3}\left(\frac{2}{3}\right)^{(q-1) / 2}
$$

which is not true for $q>67$. For primes $q, 3<q \leq 67$, the polynomial $S_{j}$ is non-zero modulo $q$. Theorem 1 is proved.

Acknowledgements. The author thanks the referee for the important remarks to the proof of Lemma 1. 


\section{References}

[1] E. R. Hansen, A Table of Series and Products, Prentice-Hall, 1973.

[2] S. Jakubec, Divisibility of the class number $h^{+}$of the real cyclotomic fields of prime degree l, Math. Comp. 67 (1998), 369-398.

[3] -, On divisibility of $h^{+}$by the prime 3, Rocky Mountain J. Math. 24 (1994), 14671473.

[4] T. Lepistö, On the growth of the first factor of the class number of the prime cyclotomic field, Ann. Acad. Sci. Fenn. Ser. A I Math. 577 (1974).

[5] T. Metsänkylä, Class numbers and $\mu$-invariants of cyclotomic fields, Proc. Amer. Math. Soc. 43 (1974), 299-300.

Matematický ústav SAV

Štefánikova 49

81473 Bratislava, Slovakia

E-mail: jakubec@mat.savba.sk 